\title{
Improvement of Sperm Morphology After Surgical Varicocele Repair
}

\section{Daria Morini}

IRCCS Reggio Emilia, Italy

\section{Giorgia Spaggiari}

Azienda Ospedaliero-Universitaria Modena, Italy

Jessica Daolio

IRCCS Reggio Emilia, Italy

\section{Beatrice Melli}

IRCCS Reggio Emilia, Italy

Alessia Nicoli

IRCCS Reggio Emilia, Italy

\section{Gaetano De Feo}

IRCCS Reggio Emilia, Italy

\section{Barbara Valli}

IRCCS Reggio Emilia, Italy

Domenico Viola

IRCCS Reggio Emilia, Italy

Simona Garganico

IRCCS Reggio Emilia, Italy

Elena Magnani

IRCCS Reggio Emilia, Italy

\section{Annalisa Pilia}

IRCCS Reggio Emilia, Italy

Alessandra Polese

IRCCS Reggio Emilia, Italy

\section{Rossana Colla}

IRCCS Reggio Emilia, Italy

\section{Manuela Simoni}

University of Modena and Reggio Emilia

\section{Lorenzo Aguzzoli}

IRCCS Reggio Emilia, Italy

\section{Maria Villani}

IRCCS Reggio Emilia, Italy

Daniele Santi ( $\nabla$ daniele.santi@unimore.it ) 
Unit of Endocrinology, Department of Medical Specialties, Azienda Ospedaliero-Universitaria of Modena, Ospedale Civile of Baggiovara, via Giardini 1355, 41126 Modena, Italy.

\section{Research Article}

Keywords: Semen analysis, sperm morphology, varicocele, varicocelectomy, male infertility

Posted Date: January 18th, 2021

DOl: https://doi.org/10.21203/rs.3.rs-145014/v1

License: (c) (i) This work is licensed under a Creative Commons Attribution 4.0 International License. Read Full License 


\section{Abstract}

Setting: A causative relationship between varicocele and impairment of semen quality has been largely investigated in the context of male infertility, although its clinical benefit remains controversial.

Objective: To investigate the effect of varicocele correction on detailed morphologic microscopic semen parameters in a large homogeneous cohort of patients and to evaluate which factors could predict semen improvement after the surgical treatment.

Design: An observational, retrospective cohort study was carried out including all patients undergoing surgical treatment for varicocele from September 2011 to March 2020 in the same clinical centre. Enrolled males performed at least one semen analysis before and one after surgical varicocele correction. Primary outcome was the detailed morphologic microscopic sperm evaluation. Secondary outcomes were conventional semen analyses.

Results: 121 males (mean age 24.6 $\$ 6.1$ years) were enrolled. Using detailed morphologic microscopic sperm evaluation, a significant morphological improvement was recorded, with a reduction in head and tail abnormalities. Moreover, a significant increase in sperm concentration $(p=0.015)$ and percentage of progressive and total motility $(p=0.022$ and $p=0.039)$ were observed after surgery. The multivariate logistic analysis identified the ultrasonography varicocele degree before surgery as a main predictor of the sperm concentration improvement $(p=0.016)$, with the highest improvement for varicocele of I and II degree.

Conclusion: For the first time, the detailed morphologic microscopic sperm evaluation highlights a relevant reduction in sperm abnormalities after varicocele surgery, showing its potential application in clinical practice.

\section{Introduction}

Varicocele is a frequent clinical condition among adult males (i.e. $15-20 \%)^{1,2}$, characterized by the detection of abnormally dilated tortuous veins within the pampiniform plexus ${ }^{3,4}$. This incidence is even more prevalent evaluating cohorts of infertile men, reaching $35-40 \%{ }^{1,2}$. Thus, not surprisingly, the association between varicocele and male infertility has been largely investigated by both clinicians and researchers dealing with couple infertility, obtaining inconclusive results. Among the suggested potential mechanisms linking varicocele to infertility, the most accredited are (i) increase of reactive oxidative species, (ii) sperm DNA damage, (iii) increase scrotal temperature and (iv) reduction of the supply of oxygenated blood and nutrients ${ }^{5-8}$. Since all these mechanisms could lead to semen quality impairment, the varicocele-induced alteration of semen parameters is probably due to a combination, not exactly quantified, of these various insults.

Generally, varicocele develops during puberty and occurs more often on the left side for anatomic reasons 9,10 . However, its diagnosis is commonly delayed, especially in asymptomatic cases, until the man 
consults an andrologist for couple infertility. The physical examination allows to classify varicocele according to a three-graded scale ${ }^{11}$. Then, varicocele diagnosis is confirmed at ultrasonography evaluation, whereby diverse scales have been proposed for classification ${ }^{12,13}$. Several international guidelines agree in recommending surgical treatment for varicocele when it is palpable and/or if abnormal semen parameters are detected ${ }^{14}$. On the contrary, there is no consensus about which surgical approach should be considered the gold standard, leaving open a spectrum of possible procedures ranging from the ligation of the spermatic vein to the microsurgical varicocelectomy ${ }^{15,16}$.

Alongside the shared surgical indications for varicocelectomy in the context of male infertility, the effective semen parameters restoration after surgical correction constitutes a debated issue. Several studies investigated varicocelectomy efficacy on semen parameters improvement and many authors described a significant semen quality improvement after varicocelectomy ${ }^{17-20}$, whereas others did not ${ }^{21-}$ 24 . Trying to overcome these contradictory results, several meta-analyses have been published on this topic. Schauer et al. evaluated 14 studies, highlighting a significant improvement in both sperm count and motility after surgical varicocelectomy ${ }^{25}$. Similarly, Kim et al. meta-analysed seven trials, detecting a significant gain of progressive sperm motility, without any effect on sperm count and morphology 26 . Moreover, Wang et al. highlighted a reduction of sperm DNA fragmentation after surgical varicocele removal, evaluating 12 clinical trials ${ }^{27}$. However, all meta-analytical approaches in this context were burdened by an extremely high heterogeneity of the studies included and, consequently, a weak statistical power, precluding a clear assessment of the efficacy of varicocelectomy. Comprehensively, it can be reasonably assumed that surgical varicocele correction improves semen parameters only in a subgroup of patients, exerting its beneficial effect mainly on semen quality, rather than on sperm number. However, the vast majority of trials on this topic did not report an improvement in sperm morphology. Although the spectrum of sperm morphological anomalies described in the world health organization (WHO) manual is broad and detailed ${ }^{28}$, these parameters are generally reported in clinical practice as a binary variable (i.e. morphologic anomalies presence or absence). Thus, a detailed morphological microscopic sperm evaluation has never been considered as a potential measurement tool to evaluate the effect of varicocele surgical repair so far.

With this in mind, this cohort study was designed to evaluate the effect of varicocele correction on detailed morphological microscopic sperm characteristics in a large, homogeneous cohort of patients, followed at the same clinical centre. As secondary objective, our study aimed at identifying those factors able to predict the seminal response to varicocelectomy, allowing a potential cost-benefit evaluation.

\section{Materials And Methods}

\section{Study design}

An observational, retrospective clinical cohort study was carried out including patients followed from September 2011 to March 2020. All patients undergoing surgical treatment for varicocele at the Day 
Surgery of the Urology Operative Unit of the Santa Maria Nuova Hospital - IRCCS of Reggio Emilia, were evaluated. Figure 1 shows patients' selection criteria.

The following inclusion criteria were considered: (i) diagnosis of varicocele at ultrasound examination, (ii) attending surgical resolution, (iii) with at least two conventional semen analyses available, one before and one after surgery. On the contrary, we excluded patients with (i) diagnosis of varicocele without surgical indication, and/or (ii) semen analyses performed only once.

Each patient was evaluated twice, before and after surgery. At each visit varicocele was assessed by both physical examination and ultrasonography (US). In particular, the US varicocele degree was evaluated in resting conditions and after Valsalva manoeuvre, graded according to the Sarteschi's 5 -item scale ${ }^{13}$. Moreover, at each visit a semen sample was collected before and 3-12 months after surgical varicocele repair, according to the clinical practice ${ }^{29}$. When more than one semen analysis has been performed after surgery, the only one falling within 3-6 months after repair was considered ${ }^{30}$.

\section{Surgical techniques}

Surgery was performed according to three different surgical techniques. Surgical subinguinal varicocelectomy provided a 3-cm transverse skin incision over the pubic ramus just below the external ring ${ }^{31}$. The cord structures were grasped carefully with an atraumatic clamp and elevated into the wound. The external spermatic fascia was split and divided and vas deferens and its vascular bundle was isolated and preserved. All veins within the spermatic cord were ligated with absorbable ties. The inguinal varicocelectomy required a 3-4-cm incision over the lower inguinal canal and carried down to and through the Scarpa fascia to isolate the aponeurosis of the external oblique muscle ${ }^{31}$. The spermatic cord within the canal was isolated and elevated from the wound and then divided and vas deferens, perivasal vessels, testicular artery, and lymphatics were all spared, and the veins ligated. Finally, anterograde scrotal sclerotherapy was performed by Tauber technique under local anaesthesia ${ }^{32}$. The spermatic cord was isolated and suspended, the anterior pampiniform plexus was isolated and cannulated. After a phlebography of the spermatic district, the sclerotization was carried out introducing simultaneously $1 \mathrm{~mL}$ of air followed by $4 \mathrm{~mL}$ of $3 \%$ ethoxysclerol.

\section{Outcomes}

Detailed morphologic microscopic sperm evaluation was considered primary endpoint of the study. Secondary endpoints were: aspect, fluidification, viscosity, and conventional semen analysis.

\section{$\underline{\text { Semen analysis }}$}

Semen analysis was performed according to the fifth version of the WHO manual ${ }^{28}$. Semen parameters were analysed for volume, sperm count, sperm motility and sperm morphology. The microscopic, morphological sperm evaluation was performed in three consecutive steps. First, a drop of semen was placed in the center of a slide, and a second slide was placed on top to obtain two samples. Second, the 
slides were fixed in methanol for 10 minutes, stained with GIEMSA solution for 40 minutes, washed with phosphate buffer and finally air dried. Third, slides were examined with a bright field optical microscope at $1000 x$ magnification, using the immersion objective, with evaluation of about 200 spermatozoa in two successive determinations. Once the difference between the two counts was greater than $10 \%$, the evaluation was repeated.

According to the WHO recommendations ${ }^{28}$, sperm morphology was evaluated in detail, considering the head, the midpiece and the tail. In particular, head anomalies include the possible presence of elongated, pyriform, duplicated, irregular, microcephaly, macrocephaly, duplicated, and/or vacuolated sperm head. Anomalies of the neck and the middle piece were described in case of asymmetrical or angled insertion of the midpiece in the head, and/or presence of abnormal excess cytoplasm larger than one third of the head size. Tail anomalies were reported in case of short, absent, curled, and/or duplicated tail 28 . Although the WHO describes the detailed morphologic microscopic sperm evaluation, this is not routinely used in clinical practice.

\section{Statistical analysis}

A first analysis was performed to describe the clinical characteristics of the patients and their varicocele. In particular, varicocele relapse was evaluated, and the comparison between pre- and post-analysis was performed excluding patients in whom a complete varicocele resolution was not achieved after surgery.

Continuous data distribution was first evaluated by Kolmogorov-Smirnov test. Normally distributed parameters were compared before and after surgery using ANOVA-univariate analysis, whereas not normally distributed parameters were compared using Man-Whitney U-test. Similarly, categorical variables were compared before and after surgery using Fisher Exact test. The number of patients with a significant improvement of sperm concentration was calculated and transformed in categorical variable. This parameter was used as dependent variable in multivariate logistic analysis, setting the following independent variables: age, varicocele degree at ultrasound, unilateral/bilateral, surgical approach and interval between surgery and semen analysis. This analysis was repeated using as dependent variables both sperm motility' and morphology's improvement after surgical treatment.

A logistic multivariate regression analysis was performed, considering all continuous variables available as covariates and categorical variables as cofactors. This analysis was performed in order to evaluate the change before and after surgery, which was considered as categorical, dependent variable. Moreover, a neural network technology was applied, using Bayesian analysis. The number of the visit, considered as pre- and post-surgery, was used as dependent variable, all continuous variables were included as covariates and all categorical variables as cofactors. The training step of the neural network randomly selected $70 \%$ of the dataset and the final model was validated on the remaining $30 \%$.

Statistical analysis was performed using the "Statistical Package for the Social Sciences" software for Windows (version 26.0; SPSS Inc., Chicago, IL, USA). For all comparisons, $p<0.05$ was considered statistically significant. 


\section{Ethical statement}

This study was conducted in accordance with the ethical standards of the Helsinki Declaration (1975, revised in 2013) and the study protocol was approved by the North Vast Area Ethics Committee (protocol number AOU 0024637/19). Considering the retrospective study design, Informed Consent was waived by the North Vast Area Ethics Committee.

\section{Results}

\section{Cohort characteristics}

One hundred and twenty-one patients were enrolled (Figure 1). The mean age at surgery was $24.6 \pm 6.1$ years.

Varicocele was detected at physical examination in $71.9 \%$ of cases (87 patients). Varicocele was unilateral (left-side) in $112(92.6 \%)$ and bilateral in 9 patients (7.4\%). At US examination, varicocele degrees were I in $6.6 \%$ (8 patients), II in $43.8 \%$ (53 patients), III in $38.8 \%$ (47 patients), and IV in $10.7 \%$ of cases (13 patients).

The surgical intervention applied was not homogeneous throughout the series. In particular, the inguinal approach phlebotomy was selected for 25 patients (20.7\%), the sub-inguinal approach for 92 patients (76\%) and the percutaneous sclerosis for 4 patients (3.3\%). Intriguingly, varicocele relapse was reported in 15 patients $(12.4 \%)$ at the first follow-up visit. The varicocele persistence was not significantly different among surgical approaches $(p=0.057)$. At this stage, antioxidant therapy was empirically prescribed to 5 patients $(4.1 \%)$. As reported in the methods section, both patients with varicocele relapse and those treated with anti-oxidants were excluded from the following analyses.

\section{Comparison between pre- and post-surgery}

The post-surgical semen analysis was performed after a mean of $183.7 \pm 112.5$ days since the surgery.

Considering the detailed morphologic microscopic evaluation, a significant improvement was detected. Indeed, head abnormalities showed a significant reduction, considering microcephaly $(3.3 \pm 3.6$ versus $2.2 \pm 2.9 \%, p=0.015)$, macrocephaly $(1.4 \pm 0.6$ versus $1.2 \pm 0.9 \%, p=0.043)$ and cytoplasmic appendix (1.4 \pm 0.8 versus $0.9 \pm 1.2 \%, p=0.041)$. Moreover, surgery led to a significant reduction of tails abnormalities, considering absence $(0.6 \pm 2.3$ versus $0.1 \pm 0.7, p=0.048)$ and coiled tail $(5.2 \pm 1.5$ versus $6.6 \pm 2.0$, $\mathrm{p}=0.037)$. Thus, surgical varicocele resolution leads to a significant improvement in specific morphological semen parameters.

Considering conventional semen parameters, the surgical treatment leads to a significant increase in sperm concentration $(p=0.015)$ and percentage of progressive and total motility $(p=0.022$ and $p=0.039$, respectively), with a significant decrease in the percentage of immotile sperms $(p=0.013)$ (Table 1$)$. In particular, semen concentration improved in $71.7 \%$ of patients (76 patients) $(p=0.010)$. Moreover, a 
significant reduction in spermatids' count in semen samples after surgery was recorded $(56.2 \%$ versus $39.3 \%, p=0.011$ ). Other conventional semen parameters did not significantly change after surgery (Table 1).

Subdividing patients according to unilateral/bilateral varicocele, the surgical treatment led to an improvement in sperm concentration $(p=0.028)$ and progressive and total motility $(p=0.041$ and $p=0.031$, respectively) when only unilateral varicocele has been considered. On the contrary, only progressive motility improved after surgery when bilateral varicocelectomy was performed $(p=0.024)$. However, the latter result has been obtained considering only 9 patients, limiting the statistical power of this finding.

Similarly, sub-inguinal phlebotomy led to a significant improvement in sperm concentration $(p=0.020)$, progressive and total motility ( $p=0.021$ and $p=0.015$, respectively), and immotile sperms $(p=0.012)$. On the contrary, no statistically significant differences were detected considering either the inguinal approach or the sclerectomy procedure. However, this lack of statistical significance could be explained by the relative low number of patients treated with these two different surgical approaches (i.e. 25 and 4 patients, respectively).

A logistic multivariate analysis was performed in order to comprehensively identify those parameters that changed after surgery. Considering all continuous and categorical variables in the analysis, we obtained a mathematical model that reached a statistical significance $(p=0.006)$, with a posteriori power of $89 \%$. Using this model, we confirmed that after surgery there was a simultaneous increase of sperm concentration and progressive sperm motility, together with a decrease of head abnormalities (elongated forms, microcephaly, macrocephaly, abnormal acrosome, absence of the acrosome) and other spermatogenic cells, such as spermatids' number (Table 2). This result was further evaluated by neural network analysis. The final model identified 2 hidden layers activated by the hyperbolic tangent able to describe the $94.1 \%$ of the dataset. The normalized relevance showed that $21.5 \%$ of the model was described by sperm concentration, suggesting that surgical varicocele repair first improves sperm concentration (Figure 2). Interestingly, the two further variables entering the model were normal forms (10.6\%) and irregular head (8.6\%) (Figure 2), suggesting that the surgical treatment significantly improve the semen quality in terms of semen morphology, considering also non-conventional and microscopic variables, such as the sperm head characteristics.

\section{Predictive variables of semen improvement}

In order to evaluate which parameters could influence semen improvement after surgical treatment, a multivariate logistic analysis was performed as described in the Materials and Methods paragraph. In this setting, only ultrasound varicocele degree before surgery significantly predicted the improvement of sperm concentration $(\mathrm{p}=0.016)$. In particular, the number of patients with improved semen parameters was higher for varicocele of I and II degree (62.5 and 67.9\%, respectively) than patients with III and IV degree (49.6 and $50.8 \%$, respectively) $(p=0.008)$. This result suggests that the surgical approach obtained better results for milder varicocele compared to severe forms, in terms of sperm concentration increase. Similarly, this result suggests that the patient's age, as well as the surgical approach or the 
unilateral/bilateral varicocele, do not influence the final improvement of semen parameters. Interestingly, these results were confirmed setting either sperm motility or morphology improvement as dependent variables.

\section{Discussion}

Surgical varicocele repair improves seminal parameters in terms of sperm number and motility (both progressive and total). Although the sperm morphology seems not to be influenced by the surgical correction if reported as a binary variable (i.e. normal or abnormal), a more detailed microscopic morphologic sperm evaluation highlights a relevant reduction in cell abnormalities.

The literature dealing with couple infertility treatments is burdened by the identification of the most appropriate end-point. Although pregnancy and live birth rates are clearly the strongest end-points in this setting, semen parameters remain the most reliable variable to evaluate the efficacy of a male factor treatment, including varicocele correction. Accordingly, many original articles measured the clinical benefit induced by varicocele surgical correction in male infertility through the potential improvement in conventional semen analysis parameters ${ }^{25,33}$. However, no studies so far applied the detailed morphologic microscopic sperm evaluation as primary endpoint of the surgical treatment. Indeed, our results demonstrated for the first time a microstructural sperm improvement after surgery, sustained by a reduction in head (i.e. microcephaly, macrocephaly and cytoplasmic appendix) and tail (i.e. agenesis and coiled form) abnormalities. Albeit the detailed microscopic morphological evaluation is not commonly applied in both clinical and research fields, microscopic sperm alterations have been described in patients carrier of varicocele ${ }^{34}$. Therefore, our results confirm the potential capability of varicocele treatment to ameliorate semen quality, highlighting a novel parameter able to measure it. Thus, our study demonstrates once again the potential semen improvement after surgical varicocele repair, with a specific novel focus on sperm morphology.

In this study, the clinical effect of the varicocele surgical treatment was analysed with a more complex statistical approach than those used so far, including different parameters obtainable in clinical practice, and trying to overcome the limitations imposed by conventional semen analysis ${ }^{35}$. Indeed, more elaborate statistical approaches would be useful to analyse an outcome that could be described or measured with different variables (i.e. surgical varicocele correction efficacy measured through sperm number, motility, morphology (binary), morphology (detailed), fructose, citric acid etc.) ${ }^{36,37}$. Thus, we applied both logistic multivariate regression analysis and neural networks confirming the clinical efficacy of surgical restoration. Regardless of the complexity of the analysis used, when all available parameters entered into the model, it is confirmed that varicocelectomy improves both the quantity (sperm concentration) and the quality (head and tail abnormalities) of semen. In addition, this statistical model was applied also to identify potential predictive variables of surgery efficacy. In our series, the semen improvement is predicted by varicocele US degree. Intriguingly, better results are obtained when milder varicocele is diagnosed at baseline, as previously suggested in a meta-analysis evaluating only sub- 
clinical varicocele ${ }^{26}$. On the contrary, the patient's age seems not to be related with the post-surgery seminal improvement. This result is in contrast with other reports ${ }^{38,39}$ but, probably, in our setting the duration of varicocele is a more a relevant parameter than age at surgery.

In our cohort, seminal improvement is detected only in patients treated with sub-inguinal flebectomy. This is contrast with previous studies highlighting a substantial overlap among surgical procedures ${ }^{40-42}$. However, our results could be due to the predominance of patients treated with the sub-inguinal surgical access ( $76 \%$ of the series). The surgical procedure presented a relapse rate of $12.4 \%$ (15 cases on 121 ) in our casuistry, independently of the technique applied. This rate is in line with data available in the literature ${ }^{43}$.

Our study has several limits, such as the retrospective study design and the imbalance among surgical approaches. Indeed, the study does not provide a case-control approach to compare the surgical techniques. Moreover, the limited availability of patients' clinical data prevented a more comprehensive predictive analysis. Finally, not all patients underwent varicocelectomy in the context of an assisted fertilization process, so data on post-surgery pregnancies is not available.

The strength of our study is the sample size. Indeed, previous trials on the same topic enrolled an average of 41 patients (minimum: 19, maximum: 82). Moreover, our case series was homogeneous, since all males were managed in the same clinical centre, with semen analyses performed in the same high-level laboratory. Finally, the use of detailed morphologic semen evaluation represents a point of strength and innovation, since it was not considered so far as an outcome of the surgery.

\section{Conclusions}

The detailed morphologic sperm evaluation has been identified as a novel parameter expressing the postsurgical semen amelioration after varicocelectomy. Moreover, milder varicocele US degrees are expected to obtain better results in terms of semen parameters improvement. This result has clinical implications, since it could help to select those patients in which the dilemma 'treat or not treat' occurs.

\section{Declarations}

Acknowledgments: Not applicable.

\section{Author contributions}

Conceptualization: DM, GS, MTV, DS; patients' enrolment: DM, JD, BM, AN, GDF, BV, DV; data collection: $D M, J D, B M, A N, G D F, S G, E M, A P, A P, R C$; statistical analysis: GS, DS; draft the article: GS, DS; revision of the article: DM, GS, JD, BM, AN, GDF, BV, DV, SG, EM, AP, AP, RC, MS, LA, MTV, DS.

Competing Interests Statement: The corresponding author states the absence of any conflict of interest for all author involved in the present study. 


\section{References}

1 Baazeem, A. et al. Varicocele and male factor infertility treatment: a new meta-analysis and review of the role of varicocele repair. European urology60, 796-808, doi:10.1016/j.eururo.2011.06.018 (2011).

2 Kupis, Ł., Dobroński, P. A. \& Radziszewski, P. Varicocele as a source of male infertility - current treatment techniques. Central European journal of urology68, 365-370, doi:10.5173/ceju.2015.642 (2015).

3 Razi, M. et al. Varicocele and Oxidative Stress: New Perspectives from Animal and Human Studies. Andrology, doi:10.1111/andr.12940 (2020).

4 Kohn, T. P., Kohn, J. R. \& Pastuszak, A. W. Varicocelectomy before assisted reproductive technology: are outcomes improved? Fertility and sterility108, 385-391, doi:10.1016/j.fertnstert.2017.06.033 (2017).

5 Merla, A., Ledda, A., Di Donato, L., Di Luzio, S. \& Romani, G. L. Use of infrared functional imaging to detect impaired thermoregulatory control in men with asymptomatic varicocele. Fertility and sterility 78 , 199-200, doi:10.1016/s0015-0282(02)03155-2 (2002).

6 Hauser, R., Paz, G., Botchan, A., Yogev, L. \& Yavetz, H. Varicocele: effect on sperm functions. Human reproduction update7, 482-485, doi:10.1093/humupd/7.5.482 (2001).

7 Blumer, C. G. et al. Sperm nuclear DNA fragmentation and mitochondrial activity in men with varicocele. Fertility and sterility90, 1716-1722, doi:10.1016/j.fertnstert.2007.09.007 (2008).

8 Sakamoto, Y., Ishikawa, T., Kondo, Y., Yamaguchi, K. \& Fujisawa, M. The assessment of oxidative stress in infertile patients with varicocele. BJU internationa/101, 1547-1552, doi:10.1111/j.1464410X.2008.07517.x (2008).

9 Masson, P. \& Brannigan, R. E. The varicocele. The Urologic clinics of North America41, 129-144, doi:10.1016/j.ucl.2013.08.001 (2014).

10 Sheehan, M. M., Ramasamy, R. \& Lamb, D. J. Molecular mechanisms involved in varicocele-associated infertility. Journal of assisted reproduction and genetics31, 521-526, doi:10.1007/s10815-014-0200-9 (2014).

11 Dubin, L. \& Amelar, R. D. Varicocele size and results of varicocelectomy in selected subfertile men with varicocele. Fertility and sterility21, 606-609, doi:10.1016/s0015-0282(16)37684-1 (1970).

12 Foroughi, A. A. et al. Clinical grading and color Doppler ultrasonography-based grading of varicocele: how compatible are the two grading systems? World journal of urology37, 1461-1465, doi:10.1007/s00345-018-2528-8 (2019).

13 Pauroso, S. et al. Varicocele: Ultrasonographic assessment in daily clinical practice. Journal of ultrasound14, 199-204, doi:10.1016/j.jus.2011.08.001 (2011). 
14 Report on varicocele and infertility: a committee opinion. Fertil Steri/102, 1556-1560, doi:10.1016/j.fertnstert.2014.10.007 (2014).

15 Cayan, S., Kadioglu, T. C., Tefekli, A., Kadioglu, A. \& Tellaloglu, S. Comparison of results and complications of high ligation surgery and microsurgical high inguinal varicocelectomy in the treatment of varicocele. Urology55, 750-754, doi:10.1016/s0090-4295(99)00603-2 (2000).

16 Al-Kandari, A. M., Shabaan, H., Ibrahim, H. M., Elshebiny, Y. H. \& Shokeir, A. A. Comparison of outcomes of different varicocelectomy techniques: open inguinal, laparoscopic, and subinguinal microscopic varicocelectomy: a randomized clinical trial. Urology69, 417-420, doi:10.1016/j.urology.2007.01.057 (2007).

17 Bertolla, R. P. et al. Sperm nuclear DNA fragmentation in adolescents with varicocele. Fertility and sterility85, 625-628, doi:10.1016/j.fertnstert.2005.08.032 (2006).

18 Yamamoto, M., Tsuji, Y., Ohmura, M., Hibi, H. \& Miyake, K. Comparison of artery-ligating and arterypreserving varicocelectomy: effect on post-operative spermatogenesis. Andrologia27, 37-40, doi:10.1111/j.1439-0272.1995.tb02093.x (1995).

19 Samplaski, M. K., Lo, K. C., Grober, E. D., Zini, A. \& Jarvi, K. A. Varicocelectomy to "upgrade" semen quality to allow couples to use less invasive forms of assisted reproductive technology. Fertility and sterility108, 609-612, doi:10.1016/j.fertnstert.2017.07.017 (2017).

20 Elbendary, M. A. \& Elbadry, A. M. Right subclinical varicocele: how to manage in infertile patients with clinical left varicocele? Fertility and sterility92, 2050-2053, doi:10.1016/j.fertnstert.2009.05.069 (2009).

21 Grasso, M. et al. Low-grade left varicocele in patients over 30 years old:the effect of spermatic vein ligation on fertility. BJU internationa/85, 305-307, doi:10.1046/j.1464-410x.2000.00437.x (2000).

22 Unal, D., Yeni, E., Verit, A. \& Karatas, O. F. Clomiphene citrate versus varicocelectomy in treatment of subclinical varicocele: a prospective randomized study. International journal of urology : official journal of the Japanese Urological Association8, 227-230, doi:10.1046/j.1442-2042.2001.00289.x (2001).

23 Zheng, Y. Q. et al. Efficacy of bilateral and left varicocelectomy in infertile men with left clinical and right subclinical varicoceles: a comparative study. Urology73, 1236-1240, doi:10.1016/j.urology.2008.11.050 (2009).

24 Nilsson, S., Edvinsson, A. \& Nilsson, B. Improvement of semen and pregnancy rate after ligation and division of the internal spermatic vein: fact or fiction? British journal of urology51, 591-596, doi:10.1111/j.1464-410x.1979.tb03609.x (1979).

25 Schauer, I., Madersbacher, S., Jost, R., Hübner, W. A. \& Imhof, M. The impact of varicocelectomy on sperm parameters: a meta-analysis. The Journal of urology187, 1540-1547, doi:10.1016/j.juro.2011.12.084 (2012).

Page 12/18 
$26 \mathrm{Kim}, \mathrm{H}$. J. et al. Clinical significance of subclinical varicocelectomy in male infertility: systematic review and meta-analysis. Andrologia48, 654-661, doi:10.1111/and.12495 (2016).

27 Wang, Y. J., Zhang, R. Q., Lin, Y. J., Zhang, R. G. \& Zhang, W. L. Relationship between varicocele and sperm DNA damage and the effect of varicocele repair: a meta-analysis. Reproductive biomedicine online25, 307-314, doi:10.1016/j.rbmo.2012.05.002 (2012).

28 WHO. WHO laboratory manual for the examination and processing of human semen. 5 th edn, (2010).

29 Ghaed, M. A., Makian, S. A., Moradi, A., Maghsoudi, R. \& Gandomi-Mohammadabadi, A. Best time to wait for the improvement of the sperm parameter after varicocelectomy: 3 or 6 months? Archivio italiano di urologia, andrologia : organo ufficiale [di] Societa italiana di ecografia urologica e nefrologica / Associazione ricerche in urologia92, doi:10.4081/aiua.2020.3.259 (2020).

30 Freeman, S. et al. Ultrasound evaluation of varicoceles: guidelines and recommendations of the European Society of Urogenital Radiology Scrotal and Penile Imaging Working Group (ESUR-SPIWG) for detection, classification, and grading. European radiology30, 11-25, doi:10.1007/s00330-019-06280-y (2020).

31 Johnson, D. \& Sandlow, J. Treatment of varicoceles: techniques and outcomes. Fertility and sterility108, 378-384, doi:10.1016/j.fertnstert.2017.07.020 (2017).

32 Ficarra, V., Sarti, A., Novara, G. \& Artibani, W. Antegrade scrotal sclerotherapy and varicocele. Asian journal of andrology4, 221-224 (2002).

33 Birowo, P., Tendi, W., Widyahening, I. S., Atmoko, W. \& Rasyid, N. The benefits of varicocele repair for achieving pregnancy in male infertility: A systematic review and meta-analysis. Heliyon6, e05439, doi:10.1016/j.heliyon.2020.e05439 (2020).

34 Chen, C. H. et al. Apoptosis and kinematics of ejaculated spermatozoa in patients with varicocele. Journal of andrology25, 348-353, doi:10.1002/j.1939-4640.2004.tb02799.x (2004).

35 Barbăroșie, C., Agarwal, A. \& Henkel, R. Diagnostic value of advanced semen analysis in evaluation of male infertility. Andrologia, e13625, doi:10.1111/and.13625 (2020).

36 Chu, K. Y. et al. Artificial Intelligence in Reproductive Urology. Curr Urol Rep20, 52, doi:10.1007/s11934019-0914-4 (2019).

37 Dave, P., Farber, N. \& Vij, S. Conventional semen analysis and advanced sperm function tests in diagnosis and management of varicocele. Andrologia, e13629, doi:10.1111/and.13629 (2020).

38 Bolat, M. S., Kocamanoglu, F., Gulsen, M., Sengul, M. \& Asci, R. The impact of age on fertility rate in patients who underwent microsurgical varicocelectomy. Andrologia51, e13234, doi:10.1111/and.13234 (2019). 
39 Mancini, M. et al. Early varicocelectomy by percutaneous scleroembolization improves seminiferous tubules spermatozoa release in the adolescent phase of testicular growth. Andrologia51, e13286, doi:10.1111/and.13286 (2019).

40 Akkoç, A. et al. Retroperitoneal high ligation versus subinguinal varicocelectomy: Effectiveness of two different varicocelectomy techniques on the treatment of painful varicocele. Andrologia51, e13293, doi:10.1111/and.13293 (2019).

41 Ameli, M., Ahmadzadeh, M., Khajavi, A. \& Nabizadeh, M. Evaluation of the success rate and complications of conventional varicocelectomy: Do we need microscopic surgery really? Urologia86, 2326, doi:10.1177/0391560318758938 (2019).

42 Bryniarski, P. et al. The comparison of laparoscopic and microsurgical varicocoelectomy in infertile men with varicocoele on paternity rate 12 months after surgery: a prospective randomized controlled trial. Andrology5, 445-450, doi:10.1111/andr.12343 (2017).

43 Çayan, S., Orhan, i., Akbay, E. \& Kadıoğlu, A. Systematic review of treatment methods for recurrent varicoceles to compare post-treatment sperm parameters, pregnancy and complication rates. Andrologia51, e13419, doi:10.1111/and.13419 (2019).

\section{Tables}

Table 1. Conventional semen parameters pre-and post-surgery. Bold value represents statistically significant difference. 


\begin{tabular}{|llll|}
\hline & Pre-surgery & Post-surgery & p-value \\
\hline pH & & & \\
\hline Semen volume (mL) & $3.1 \pm 1.3$ & $3.0 \pm 1.2$ & 0.571 \\
\hline Sperm concentration (millions/mL) & $32.6 \pm 30.6$ & $43.9 \pm 39.4$ & 0.015 \\
\hline Total sperm number (millions) & $99.0 \pm 99.5$ & $121.9 \pm 113.2$ & 0.115 \\
\hline Progressive motility (\%) & $39.1 \pm 15.5$ & $44.0 \pm 17.1$ & 0.022 \\
\hline Non-progressive motility (\%) & $11.8 \pm 7.8$ & $10.9 \pm 5.6$ & 0.350 \\
\hline Immotile sperm (\%) & $49.0 \pm 14.6$ & $44.8 \pm 16.6$ & 0.039 \\
\hline Total motility (\%) & $50.7 \pm 14.4$ & $55.8 \pm 16.8$ & 0.013 \\
\hline Normal forms (\%) & $2.02 \pm 2.1$ & $3.4 \pm 12.8$ & 0.275 \\
\hline Fructose ( $\mu$ mol/ejaculate) & $59.4+39.5$ & $55.1 \pm 35.3$ & 0.400 \\
\hline Citric acid ( $\mu$ mol/ejaculate) & $85.5 \pm 46.8$ & $88.0 \pm 50.8$ & 0.714 \\
\hline
\end{tabular}

Table 2. Logistic multivariate analysis results. For each parameter included in the regression analysis, the strength of the regression (F) and the $p$-value were reported. Bold value represents statistically significant variable. 


\begin{tabular}{|c|c|c|}
\hline & $\mathbf{F}$ & p-value \\
\hline Age at surgery & 0.172 & 0.679 \\
\hline pH & 1.201 & 0.273 \\
\hline Semen volume & 1.167 & 0.280 \\
\hline Sperm concentration & 6.334 & 0.005 \\
\hline Total sperm number & 1.100 & 0.078 \\
\hline Progressive motility & 7.499 & 0.004 \\
\hline Non-progressive motility & 0.517 & 0.472 \\
\hline Immotile sperm & 0.044 & 0.834 \\
\hline Total motility & 0.970 & 0.325 \\
\hline Normal forms & 1.410 & 0.235 \\
\hline Fructose & 0.008 & 0.931 \\
\hline Citric acid & 1.241 & 0.265 \\
\hline Elongated head & 5.881 & 0.015 \\
\hline Pyriform head & 1.032 & 0.614 \\
\hline Microcephaly & 4.389 & 0.036 \\
\hline Macrocephaly & 4.842 & 0.028 \\
\hline Duplicated head & 0.113 & 0.737 \\
\hline Irregular head & 1.642 & 0.210 \\
\hline Abnormal acrosome & 7.758 & 0.005 \\
\hline Absent head & 0.066 & 0.797 \\
\hline Acrosome agenesis & 5.568 & 0.018 \\
\hline Cytoplasmic appendix & 0.347 & 0.556 \\
\hline Absent tail & 2.388 & 0.122 \\
\hline Short tail & 0.058 & 0.810 \\
\hline Coiled tail & 1.988 & 0.159 \\
\hline Duplicated tail & 1.255 & 0.263 \\
\hline
\end{tabular}




\begin{tabular}{|lll|} 
Spermatocyte & 0.908 & 0.341 \\
\hline Spermatids & 9.400 & 0.002 \\
\hline Agglomination & 0.014 & 10.000 \\
\hline Mixed Anti-globuline Reaction (MAR) test & 0.301 & 0.583 \\
\hline Leucocytes & 2.372 & 0.066 \\
\hline
\end{tabular}

\section{Figures}

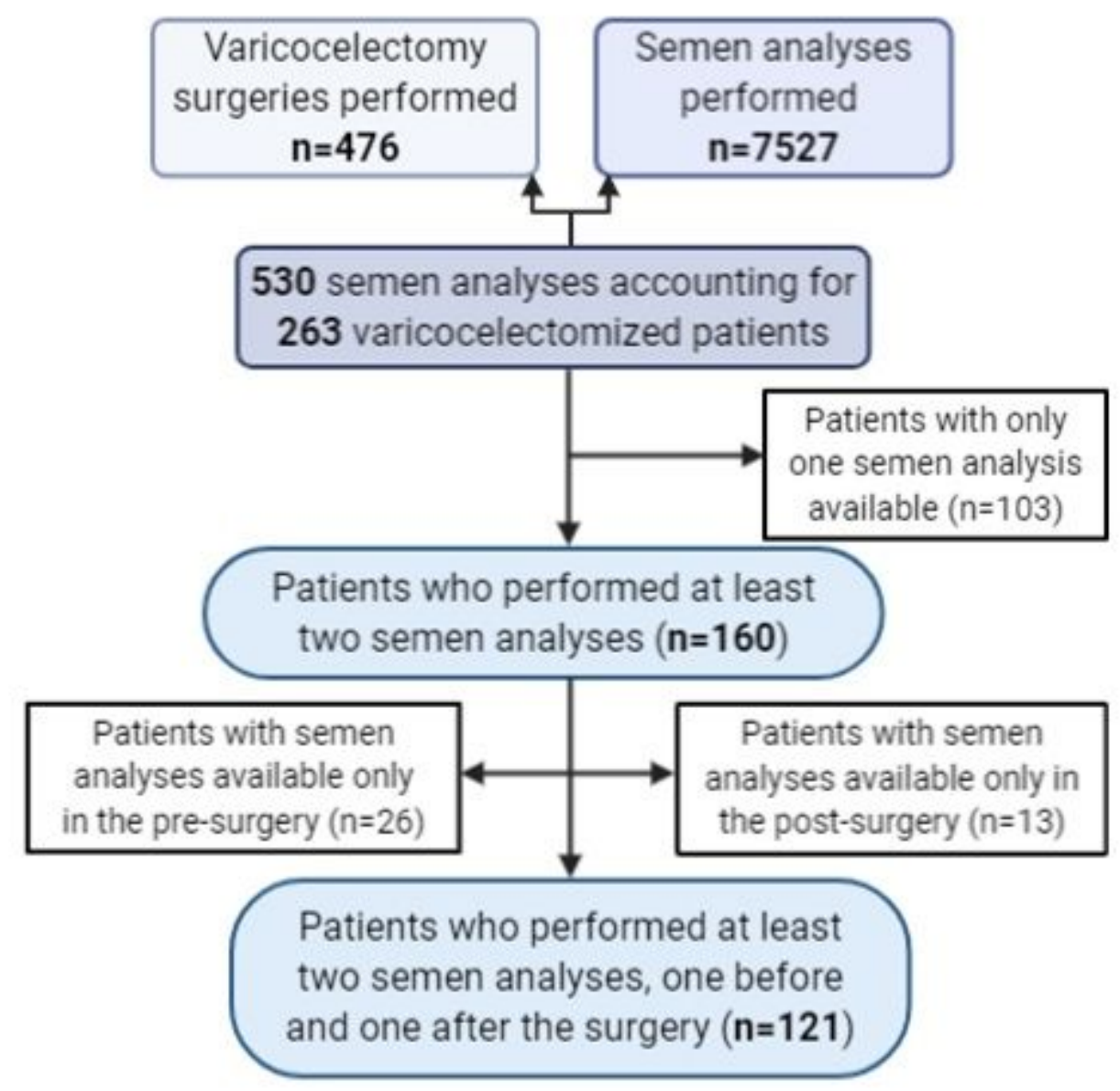

Figure 1

Flow chart of the patients' enrolment phase. 


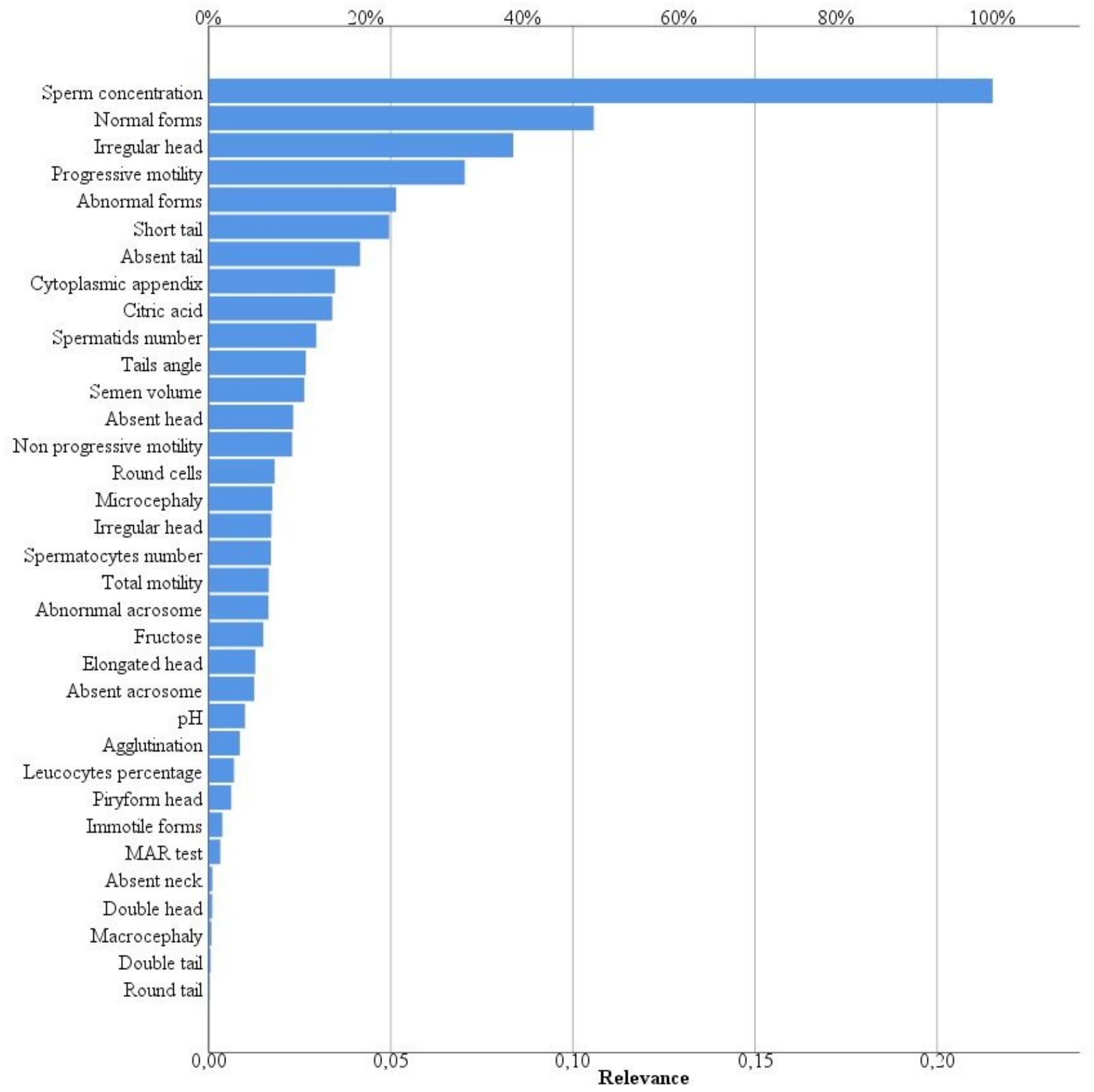

\section{Figure 2}

Neural network analysis. The graph shows the relevance of each variable included in the neural network. [Footnote to figure 2: MAR = Mixed Anti-globuline Reaction] 